\title{
Gênese e probabilidade ocorrência de tornados no Rio Grande do Sul, Brasil
}

Genesis and probability of tornado occurrence in Rio Grande do Sul, Brazil

\author{
Luana Writzl ' ; Cássio Arthur Wollmann " ; Amanda Comassetto lensse III \\ Aline Nunes da Silva IV
}

\section{RESUMO}

Com o objetivo de estudar a gênese dos tornados no Rio Grande do Sul, foi feito um banco de dados, utilizando dados de canais de meteorologia, jornais das cidades dos registros e trabalhos publicados para encontrar registros confirmados. Com auxílio das Cartas Sinóticas da Marinha do Brasil e das imagens de satélite do INPE analisadas no dia de cada evento, identificaram-se os sistemas atmosféricos que estiveram presentes no dia dos registros dos tornados. Ainda foi identificada a sazonalidade dos eventos registrados.

Palavras-chave: Eventos extremos; Histórico; Tornados; Circulação Atmosférica; RS

\section{ABSTRACT}

This research concerns in to study the genesis of tornadoes in Rio Grande do Sul, a database was made using meteorological channel data, city newspapers and published works to find confirmed records. We used Synoptic Letters of the Brazilian Navy and the INPE satellite images analyzed on the day of each event, the atmospheric systems that were present on the day of the tornado records were identified. The seasonality of recorded events was still identified.

Keywords: Extreme events; Historic; Tornadoes; Atmospheric circulation; RS

\footnotetext{
I Universidade Federal de Santa Maria, Santa Maria, Brazil. luanawritzl@yahoo.com.br

" Universidade Federal de Santa Maria, Santa Maria, Brazil.:cassio_geo@yahoo.com.br

"' Universidade Federal de Santa Maria, Santa Maria, Brazil. comassettoamanda@gmail.com

IV Universidade Federal de Santa Maria, Santa Maria, Brazil. alinendeca@hotmail.com
} 


\section{INTRODUÇÃO}

Os tornados são fenômenos atmosféricos intensos e quando atingem áreas habitadas ou com valor econômico acabam trazendo muitos transtornos assim como as chuvas fortes, acompanhadas de granizo. Nesse contexto os fenômenos tornádicos têm grandes impactos socioeconômicos, podendo ser associados às causas de desastres naturais, o que ressalta a relevância do seu estudo. Além disso, grande parte da população desconhece que o Brasil é o segundo lugar com maior incidência de tornados no mundo (BECK; VERZENHASSI 2008).

Os tornados representam ameaça potencial para atividades importantes como aviação, agricultura, transmissão e distribuição de energia elétrica (NASCIMENTO, 2005). A dinâmica da atmosfera faz parte das manifestações da natureza e ocorrerá com ou sem a presença do homem, e suas consequências podem variar. A preocupação maior a cerca desses fenômenos naturais é quando resulta em vítimas fatais e com o aumento da população seja urbana ou rural, tais eventos acabam por serem registrados mais frequentemente.

Nesse sentido, o Rio Grande do Sul frequentemente é atingido por tornados e somente para o ano de 2014 foram 13 registros, confirmando a importância de tratar o assunto no meio acadêmico, principalmente o geográfico. Alguns trabalhos já foram realizados com o tema de tornados no Brasil, como Rodrigues (2011) que estudou os tornados no centro-sul do Brasil, Candido (2012) que propõe um modelo de proposta de avaliação de danos e Reckziegel (2007) que avalia desastres naturais no Rio Grande do Sul, incluindo os tornados.

De acordo com Reckziegel (2007), os desastres naturais associados à dinâmica atmosférica estão relacionados com grandes amplitudes termo barométricas, o que provocaria a intensificação do regime dos ventos. Candido (2012) avalia que os tornados surgem porque a turbulência aliada à redução da temperatura em certos pontos no interior das nuvens propicia ocorrência de ventos rotacionais que, em condições ideais, podem afunilar e, eventualmente, tocar o solo. 


\section{CARACTERIZAÇÃO E JUSTIFICATIVA}

Os eventos atmosféricos intensos são grandes responsáveis pelas causas dos desastres naturais. Os tornados são eventos extremos e quando atingem áreas habitadas ou com valor econômico acabam trazendo muitos transtornos assim como as chuvas fortes, acompanhadas de granizo. Nesse contexto tais fenômenos climáticos têm grandes impactos socioeconômicos, e apesar da climatologia não ser uma ciência social, o estudo dos tornados pelo viés climatológico usando como ferramenta os Sistemas de Informações Geográfica (SIG) torna-se importante para entender sua distribuição e tempo de retorno desses eventos.

O tornado é definido pelo Glossário de Meteorologia (Hushcke, 1959), como uma violenta coluna de ar giratória, pendente de uma nuvem cumulonimbos, visualizado como uma nuvem em forma de funil. Fujita (1981) explica que o tornado é um mesociclone com rápida rotação, acompanhado por ventos destruidores próximo ao solo, e normalmente observado como uma nuvem funil pendente de uma nuvem mãe. Candido (2012) avalia que os tornados surgem porque a turbulência aliada à redução da temperatura em certos pontos no interior das nuvens propicia ocorrência de ventos rotacionais que, em condições ideais, podem afunilar e, eventualmente, tocar o solo.

Segundo Dyer (1994), a maior ocorrência de tornados no Brasil encontra-se nas Regiões Sul e Sudeste do país. O Rio Grande do Sul, por possuir em sua dinâmica, um clima que possui a participação de sistemas atmosféricos intertropicais e extratropicais, Wollmann; Galvani (2012) salientam que o principal fator geográfico de individualizações do clima no estado é o seu relevo/geomorfologia.

O Rio Grande do Sul é atingido por altas e baixas pressões, de acordo com Sleiman (2008) e essas, muitas vezes, estão associadas à passagem de ciclones extratropicais. Entre duas massas de ar com pressões altas, existe sempre uma zona de baixa pressão que constitui uma descontinuidade para qual convergem os ventos das duas massas de ar ou das altas pressões. Na descontinuidade, os ventos convergentes ou ciclônicos estão associados à instabilidade atmosférica com tempo instável e geralmente chuvoso. (NIMER, 1990). 
Alguns sistemas atmosféricos estão intimamente ligados aos registros de tornados no Rio Grande do Sul, como avaliou lensse (2015), sendo os principais: A Massa Tropical Continental (MTC) que produz Instabilidades Tropicais (IT) e Complexos Convectivos de Mesoescala (CCM's) e as Frente Polares com ciclogênese e sem ciclogêneses. Salienta-se que esses sistemas atmosféricos possuem muita convecção e distúrbios de pressão atmosférica, o que muda o regime dos ventos e, dessa forma a formação de supercélulas produtoras de tornados é facilitada.

Além disso, grande parte da população desconhece que o Brasil, sobretudo o Rio Grande do Sul, é o segundo lugar com maior incidência de tornados no mundo. (BECK; VERZENHASSI, 2008). A área a ser estudada encontra-se no "polígono dos Tornados" como nomeou (CANDIDO, 2012, p.65), fazendo alusão ao corredor dos tornados nos Estados Unidos. Nos últimos 18 anos foram registrados cerca de 65 tornados só no Rio Grande do Sul (Figura 1), segundo lensse (2015), fortalecendo a importância de estudos.

Figura 1 - Mapa de localização de tornados no Rio Grande do Sul para o período de análise

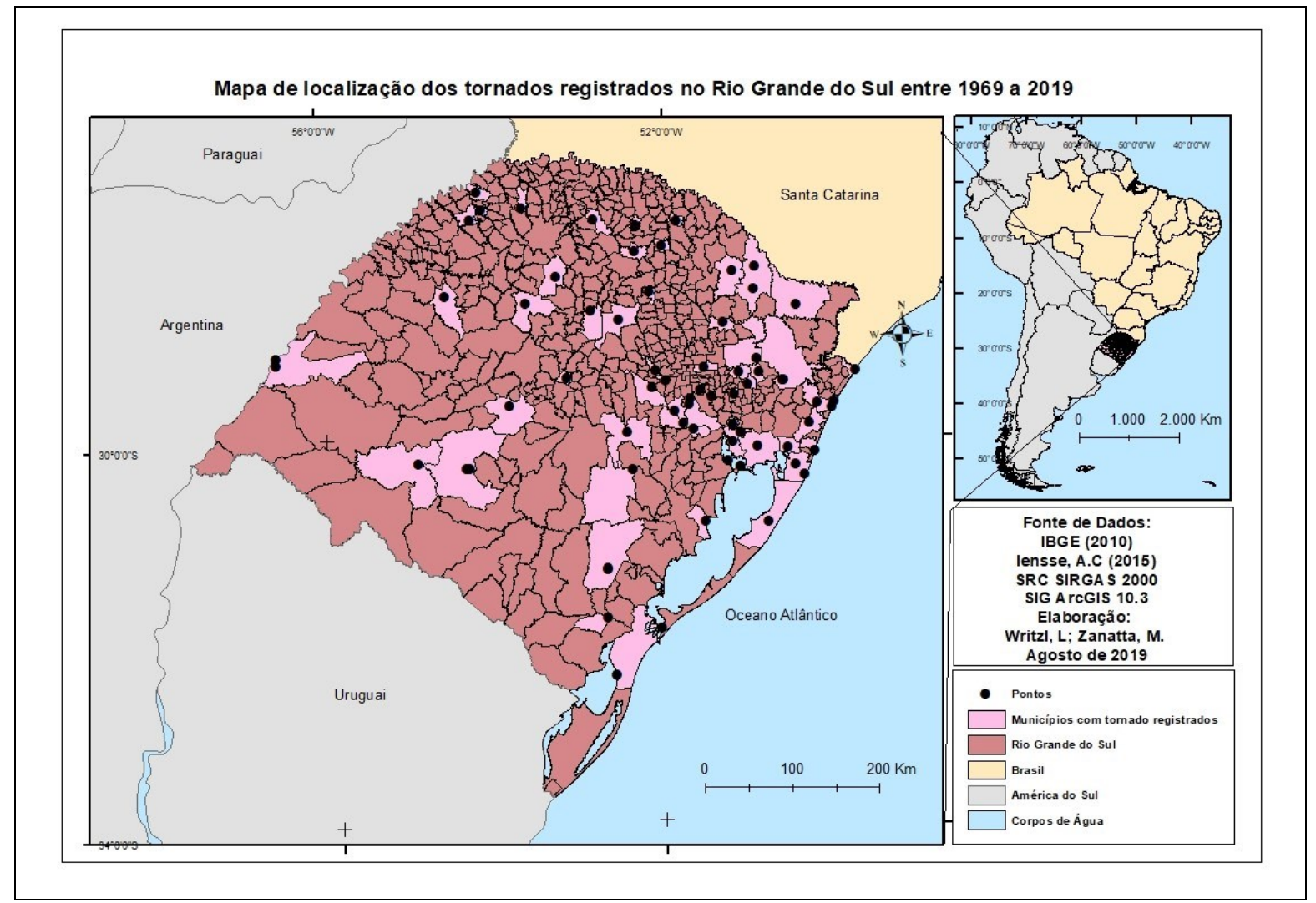


Os Tornados representam ameaça potencial para atividades importantes como, por exemplo, aviação, agricultura, transmissão e distribuição de energia elétrica (NASCIMENTO, 2005). Reckziegel (2007) coloca que a dinâmica da atmosfera faz parte das manifestações da natureza e ocorrerá com ou sem a presença do homem e suas consequências podem variar. A preocupação maior acerca desse fenômeno natural é quando resulta em vítimas fatais e com o aumento da população seja urbana ou rural, tais eventos acabam por ser registrados mais frequentemente.

\section{PROBLEMA A SER RESOLVIDO}

O registro de tornados no Rio Grande do Sul tem aumentado e com isso tornase importante um estudo acerca da sua gênese climatológica e meteorológica. Com dados de sistemas atmosféricos foi possível identificar as condições que propiciaram a formação do tornado e a partir das imagens de radar foram identificadas as assinaturas tornádicas. Dessa forma ao fazer a compilação dos dados foi possível entender como esses fenômenos acontecem no Rio Grande do Sul.

\section{OBJETIVOS E METAS}

Para compreender a climatologia dos tornados no Rio Grande do Sul, buscouse pontuar alguns objetivos específicos e metas para chegar aos resultados;

1.Construir um inventário dos eventos tornádicos ocorridos entre 1967 e 2019 no Rio Grande do Sul; minerando o maior número de dados para cada fenômeno tais como: Cidade, data, intensidade do tornado, pressão atmosférica, temperatura do ar.

2.Espacializar os eventos de tornados registrados; e analisá-los de acordo com os dados coletados no primeiro objetivo.

3.Identificar os sistemas atmosféricos atuantes antes e durante o registro; a partir das cartas sinóticas da Marinha do Brasil e as assinaturas tornádicas 
serão identificadas através de tratamento de imagens de radar coletadas do acervo da REDEMET vinculada ao comando da aeronáutica;

4.Identificar os locais com maior probabilidade de ocorrência de tornados no Rio Grande do Sul; e identificar os padrões da atmosfera antes e durante a ocorrência dos eventos, utilizando a interpolação dos dados no objetivo 3.

\section{REVISÃO BIBLIOGRÁFICA}

Stull (2000) define Tornado como um fenômeno originado a partir de uma vorticidade horizontal na baixa troposfera, próxima a superfície, ao se intensificarem inclinam-se para a posição vertical devido as correntes ascendentes de ar.

Os Tornados derivam de nuvens chamadas Cumulonimbos (Cb). Tais eventos normalmente são decorrentes de nuvens de elevado desenvolvimento vertical e intensa turbulência interna (FUJITA-PEARSON, 1971). Apesar de estar próxima a superfície terrestre, as $\mathrm{Cb}$ podem chegar a níveis muito altos na atmosfera, até o topo da troposfera.

Essas nuvens por acumular muita energia interna, às vezes causam ventos fortes, precipitação, relâmpagos e granizo aparecendo isoladas, mas quando atingem seu máximo de desenvolvimento, podem vir a ser uma supercélula com uma tempestade tornádica. Contudo, alguns tornados possam ter origem em sistemas frontais, sem a presença de supercélulas (WAKIMOTO; WILSON, 1989).

Conforme Ayoade (2003) o calor latente contido no vapor d'água é importante fonte de energia para a circulação atmosférica e para o desenvolvimento de perturbações atmosféricas. Candido (2012) analisa que quanto mais úmido o local, maior a quantidade de vapor condensado, o que permite inferir que este seja um dos motivos pelos quais muitos dos tornados em território brasileiro não possam ser visualizados.

Deve-se salientar que nem toda nuvem de tempestade apresenta tornados, mas qualquer formação de origem convectiva ou frontal que apresente condições propícias ao desenvolvimento de tornados é chamada de tempestade tornádica (ALLABY, 2004). Para o desenvolvimento da tempestade é necessária à mudança na direção dos ventos bem como o aumento da velocidade, dessa forma cria-se uma 
tendência de rotação horizontal na baixa troposfera. Essa mudança chama-se cisalhamento do vento, e permite que o ar ascendente passe da posição horizontal para a posição vertical. A formação da área de rotação possui o comprimento de 4 a 6 km e corresponde a extensão da tempestade que são fortes e violentas

A intensidade dos tornados é medida pela Escala Fujita (EF), esta escala foi desenvolvida pelo Dr. Tetsuya Theodore Fujita, em 1973, da Universidade de Chicago do centro de previsão de tempestades severas. Ao contrário do que se pensa a escala Fujita não mede os Tornados pela velocidade dos ventos nele envolvidos ou seu tamanho, mas mede de acordo com os danos que o Tornado causou de acordo com Fujita (1981).

A Figura 2 apresenta o estado do Rio Grande do Sul, no Sul do Brasil o qual foi o indicador espacial dessa pesquisa. O Rio grande do Sul faz fronteira com o Uruguai ao Sudoeste, com Argentina a Noroeste, o estado de Santa Catarina a Norte e a Leste é banhado pelo Oceano Atlântico. Além disso, o Estado é dividido em 7 mesorregiões, sendo elas: Centro Oriental, Centro Ocidental, Metropolitana, Nordeste, Noroeste, Sudeste e Sudoeste (ALONSO, 2009).

Figura 2 - Mapa de localização do Rio Grande do Sul, no sul do Brasil

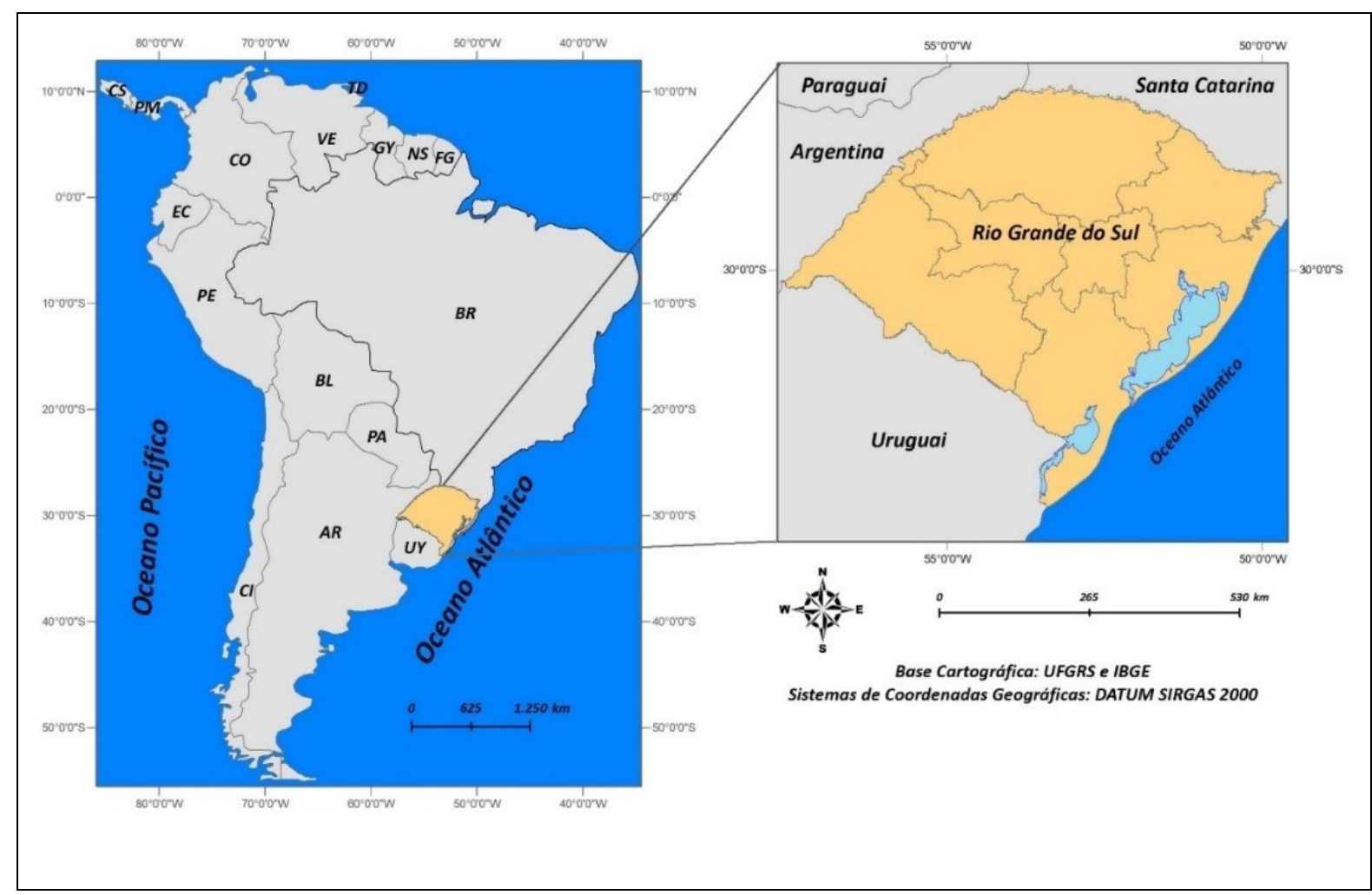




\section{METODOLOGIA}

Para atingir os objetivos da pesquisa sobre a climatologia de tornados no Rio Grande do Sul, foi necessário seguir algumas etapas que serão descritas a seguir para melhor compreensão. A primeira etapa consistiu em construir uma fundamentação teórica acerca dos tornados e como eles ocorrem em outras partes do mundo, além disso buscou-se fundamentar a climatologia do Rio Grande do Sul para construir uma base teórica-metodológica que sustente os resultados da pesquisa.

A segunda etapa foi a construção do inventário sobre os tornados registrados no Rio Grande do Sul entre 1967 e 2019. O inventário foi feito em tabela Excel e composto das seguintes variáveis: Data, local, causas e efeitos socioeconômicos. Ainda nessa etapa os registros foram especializados nas coordenadas ( $X, Y$ e $Z$ ) através da ferramenta de geoestatística de krigagem ordinária no software ArcGIS 10.3, afim de evitar sobreposição de registros sobre uma mesma coordenada.

Na terceira etapa, foram coletadas as cartas sinóticas da Marinha do Brasil, para identificar os sistemas atmosféricos que estiveram presentes no dia do registro dos tornados para compreensão da circulação atmosférica regional. Para identificar os padrões de assinaturas tornádicas, foram coletadas as imagens de radar (Santiago e Canguçu) da REDEMET, onde é possível acompanhar os dados minuto a minuto do desenvolvimento das nuvens.

A quarta e última etapa foi para a construção de mapas de probabilidade de ocorrência utilizando interpolação de dados, para ponderar os resultados. A construção foi feita no software ArcGIS 10.3.

\section{RESULTADOS}

No período de 1967 a 2019 foram registradas 67 ocorrências de tornados no Rio Grande do Sul. Entre eles, 22 ocorreram na primavera, 17 no verão, 11 no outono e 17 no inverno. Foi possível perceber um aumento significativo entre 2006 e 2008, 
assim como o ano de 2014, que foi o ano com mais registros de tornados no Rio Grande do Sul.

Figura 3 - Número de tornados por ano de ocorrência

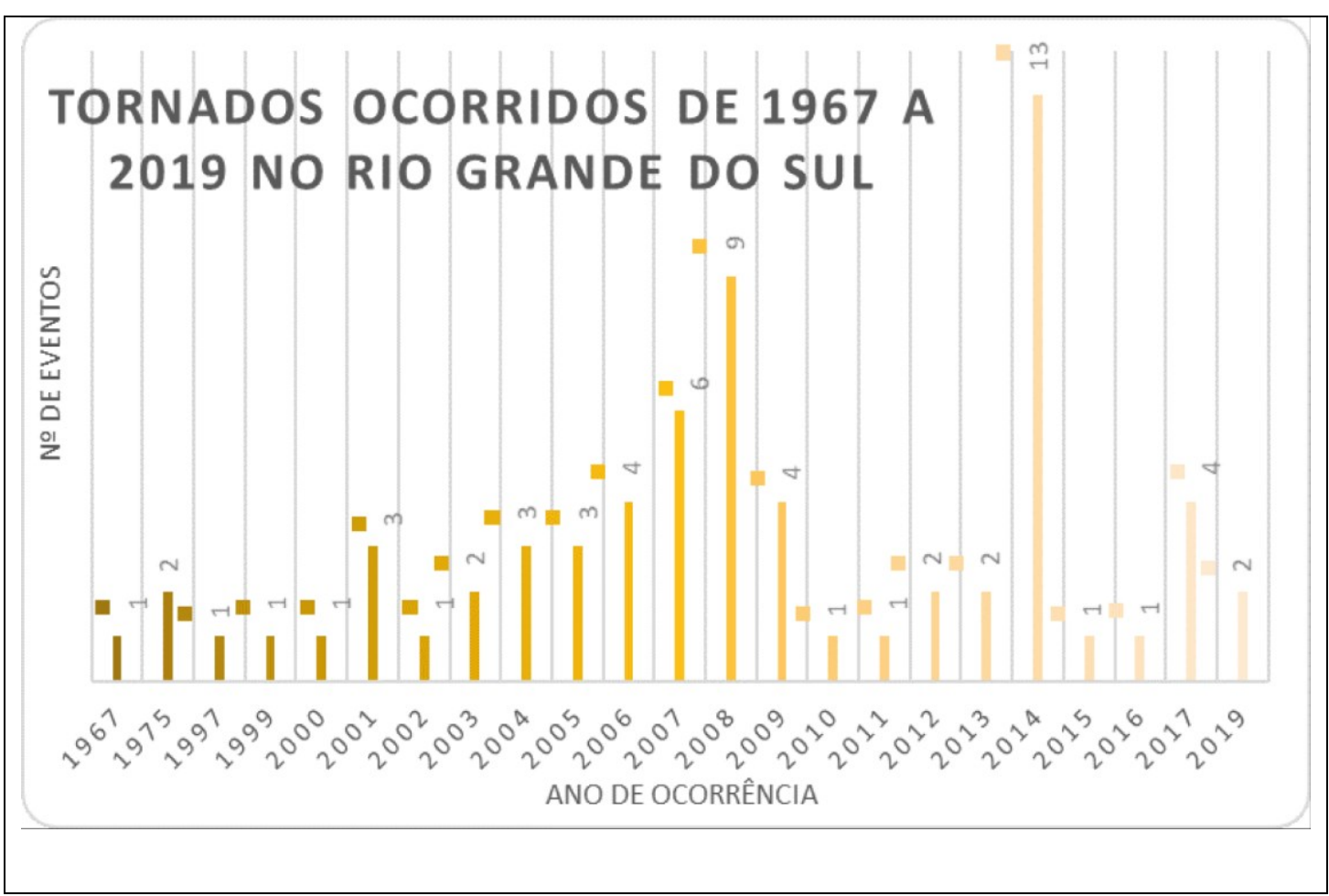

Org.: Writzl, L. (2019)

Figura 4 - Quantidade de tornados por sazonalidade

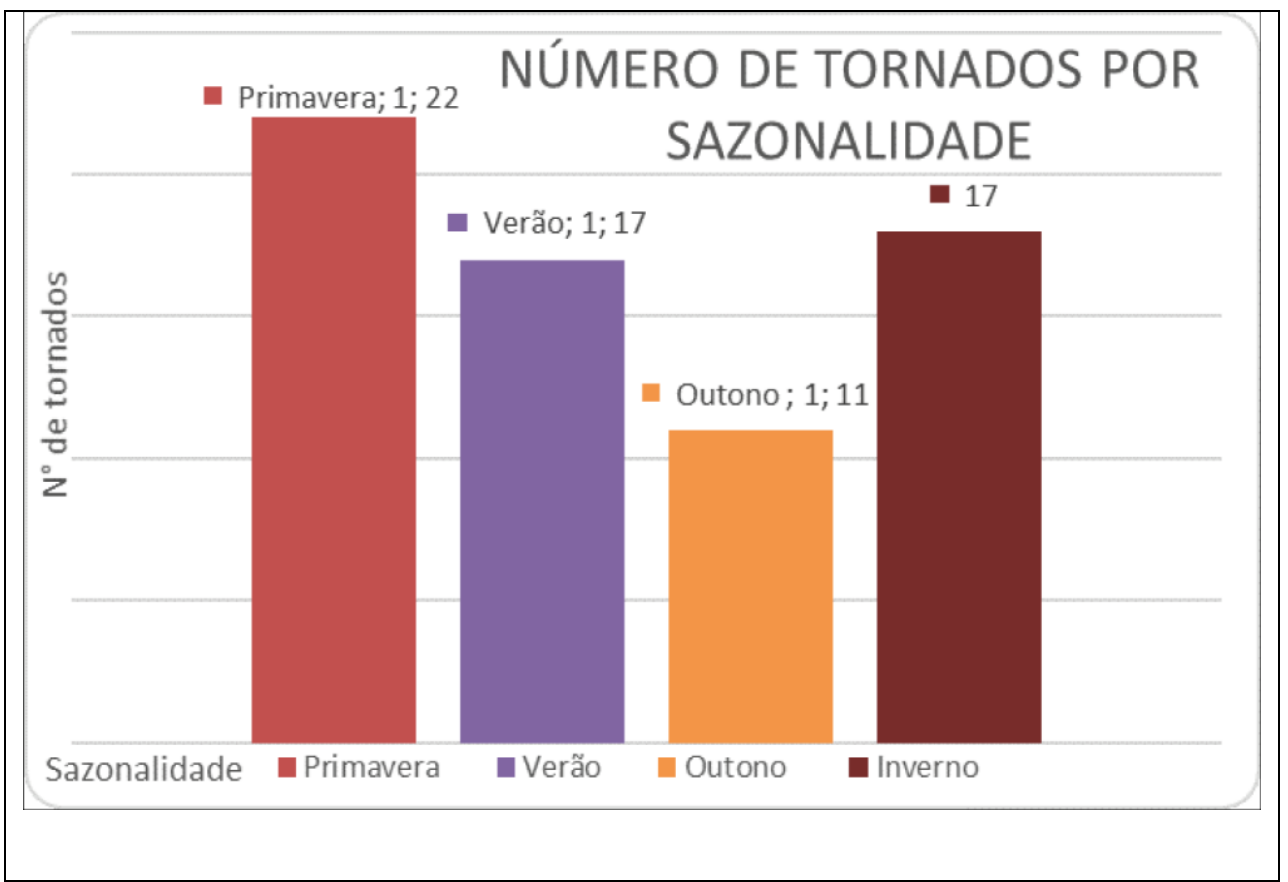

Org.: Writzl, L. (2019). 
Os registros, em sua maioria, foram realizados por moradores e noticiados pelas mídias locais, tais como, televisão, jornais, internet, rádio, entre outros. Através do Mapa da Densidade de Kernel (figura 5), foi possível analisar a densidade de ocorrência dos tornados no Rio Grande do Sul para o período de análise.

Figura 5 - Densidade das ocorrências de tornados no Rio Grande do Sul no período de 1967 a 2019

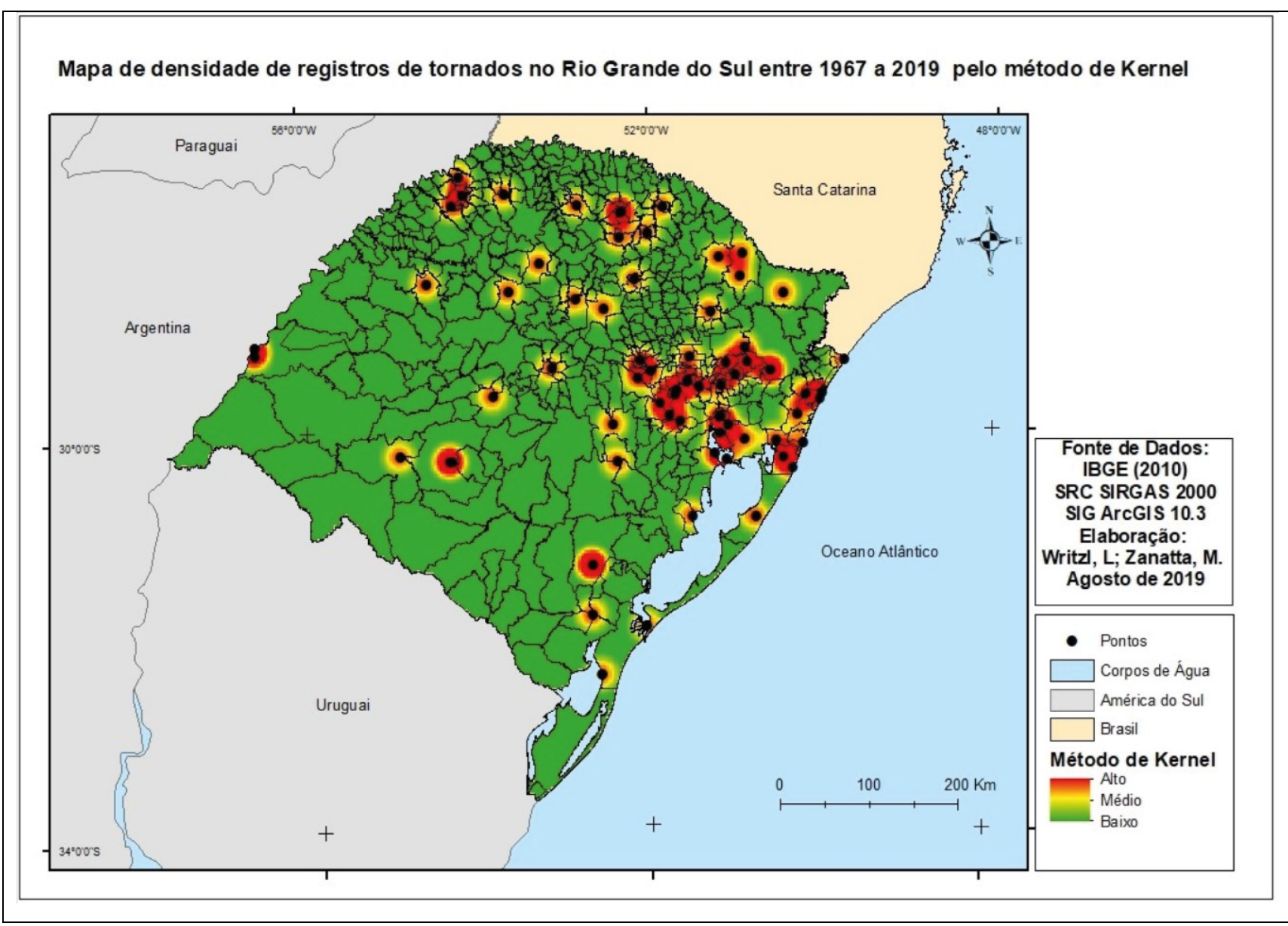

De acordo com a Figura 5, foi possível observar que houve uma densidade maior de ocorrências de tornados na região metropolitana do estado, que tem como característica as pequenas taxas de elevações de altitudes comparada ás demais regiões. A principal causa para essa discrepância espacial de ocorrência é devido a quantidade de população que ali reside e consequentemente o acesso à tecnologia e a facilidade aos meios de informações que a região é beneficiada, aumentam a demanda de registros. O crescimento de ocorrências em 2007, 2008 e 2014 também 
podem ser explicados por esse fator, pois a maioria desses registros foram próximos da região metropolitana, ou localizadas nela, assim como em munícipios mais populosos comparados aos demais.

Dessa forma, a tendência de aparecimento de tornados possivelmente continuará nessas regiões, mas isso não significa que não exista ocorrência de tornados em outras partes do estado, frequentemente.

Figura 6 - Mapa de influência dos tornados para o período de análise

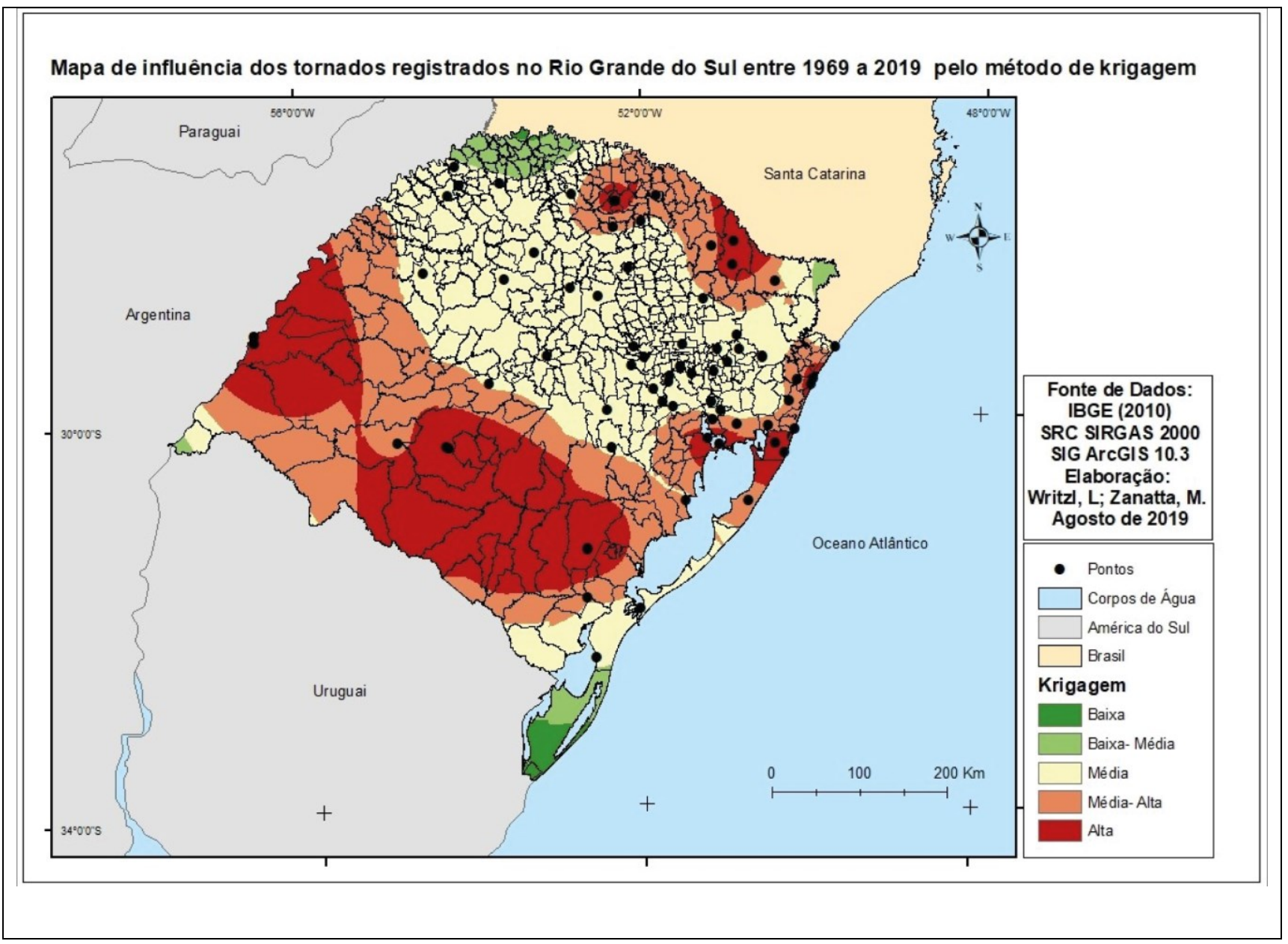

De acordo com o mapa de Krigagem (figura 6), relacionado aos tornados que foram registrados, é possível perceber a área de influência que foi encontrada pelo método, assim é viável analisar quais são as probabilidades de ocorrências de tornados para o Rio Grande do Sul de acordo com as estimativas da distribuição dos tornados para o estado. Foi possível perceber que em alguns municípios houve mais de uma ocorrência de tornados, dessa forma, com a interpolação de dados foi 
possível perceber em vermelho as altas taxas de ocorrências. Nos extremos Norte e Sul do estado, há baixas probabilidades de ocorrências, nessas áreas, se localizam municípios com baixo índice populacional comparados aos demais, o que colabora para a inexistência dos registros.

Os principais sistemas atmosféricos que atuaram nas ocorrências registradas foram em sua maioria por Frente Polar Atlântica e Ciclone como mostra a Figura 7.

Figura 7 - Sistemas atmosféricos atuantes no período de análise

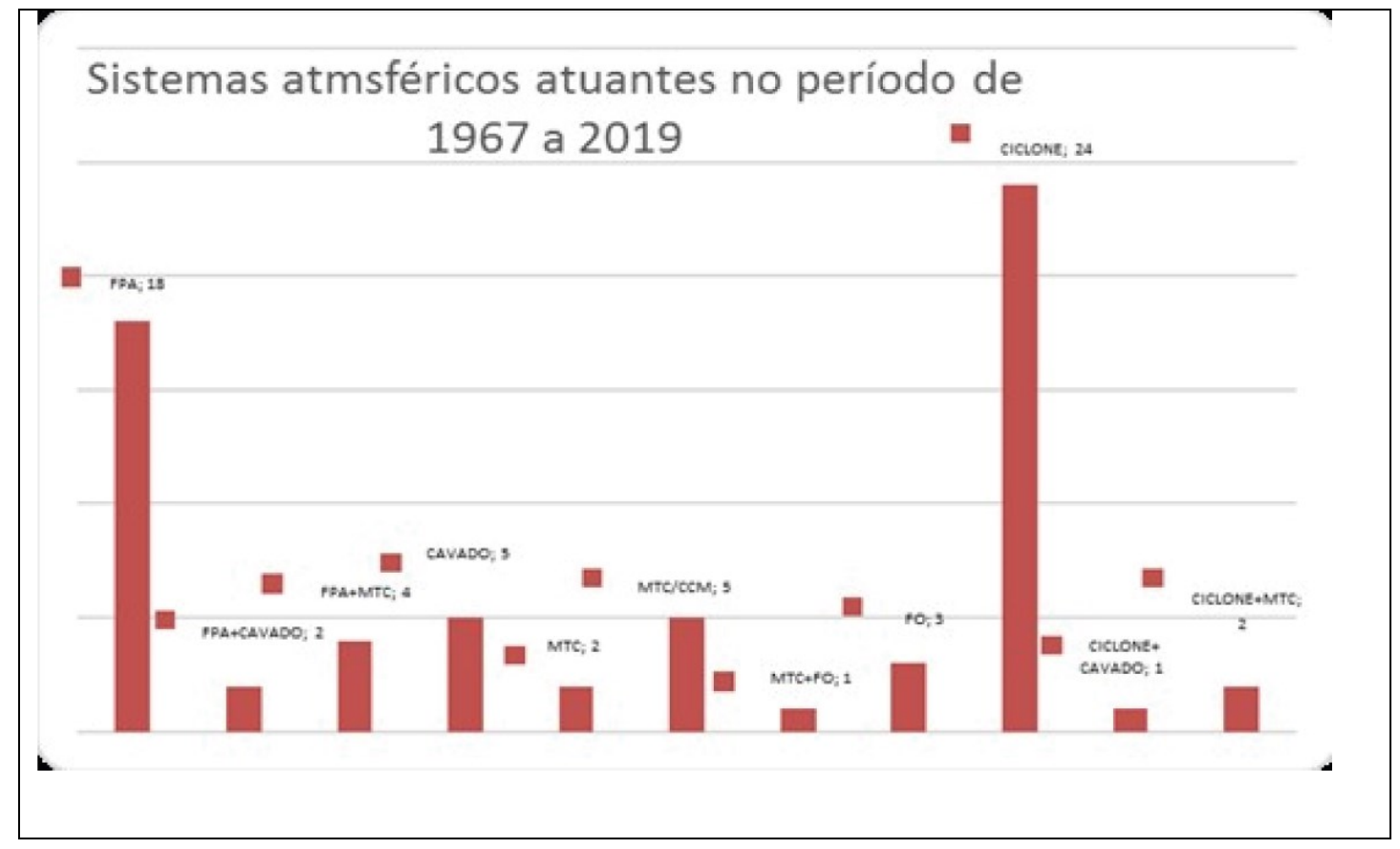

Org.: Writzl, L. (2019)

Foi possível observar os diferentes sistemas atmosféricos atuantes nas distintas estações do ano. Como mostram as Figuras 8 e 9 que representam o inverno e o verão respectivamente, foi possível perceber que a FPA foi o principal sistema atmosférico que estava atuando no período de análise, a trajetória da frente polar atlântica depende da estação do ano, o que explica sua atuação e sua relação com as instabilidades. No inverno sua atuação fica ainda mais proeminente. 
Figura 8 - Sistemas atmosféricos registrados no inverno

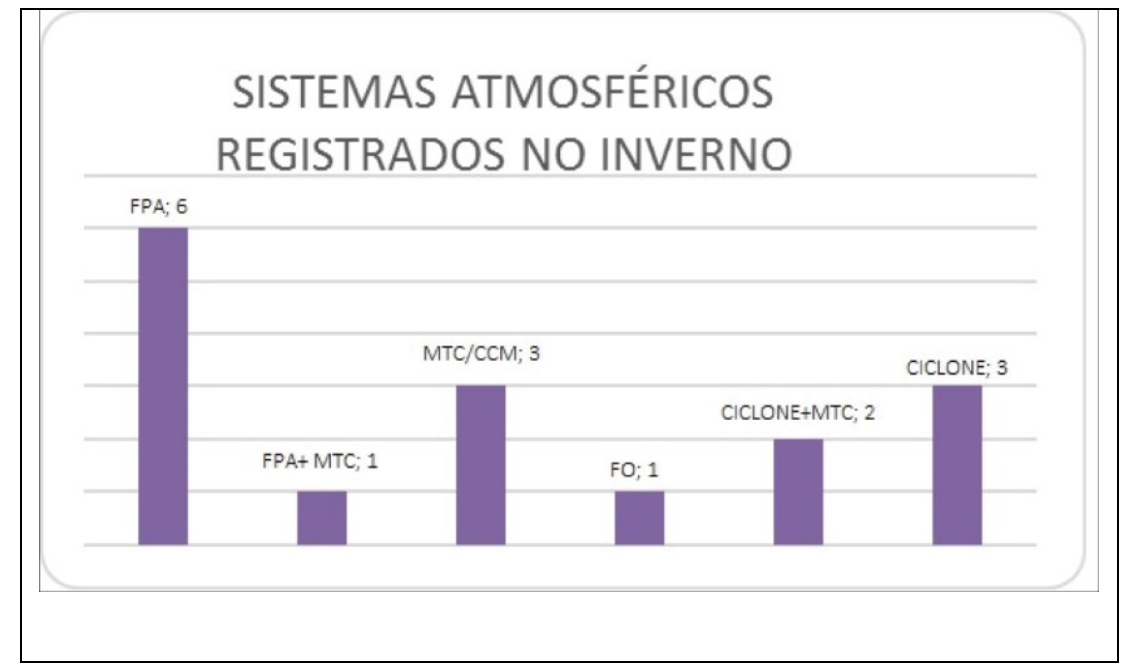

Org.: Writzl, L. (2019)

Figura 9 - Sistemas Atmosféricos registrados no verão

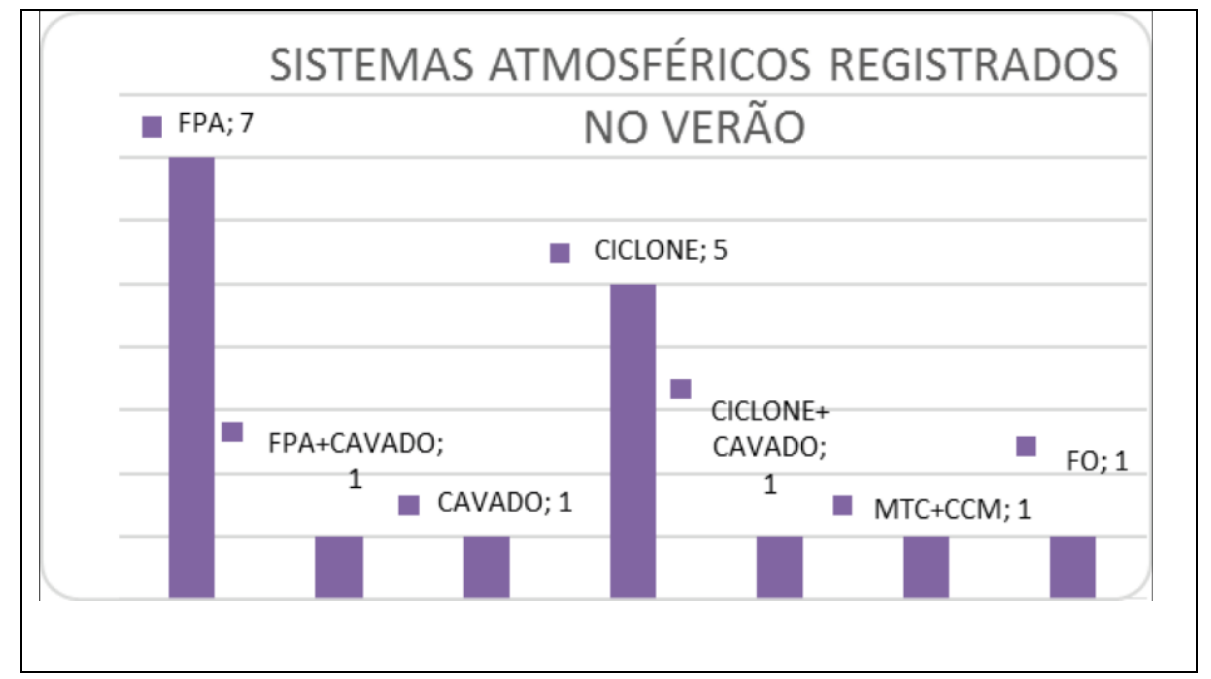

Org.: Writzl, L. (2019)

Durante a primavera e o outono, ficou evidente a atuação de centros de baixas pressões na grande maioria dos eventos, como mostra a Figura 10 e 11 respectivamente. 
Figura 10 - Sistemas Atmosféricos registrados na primavera

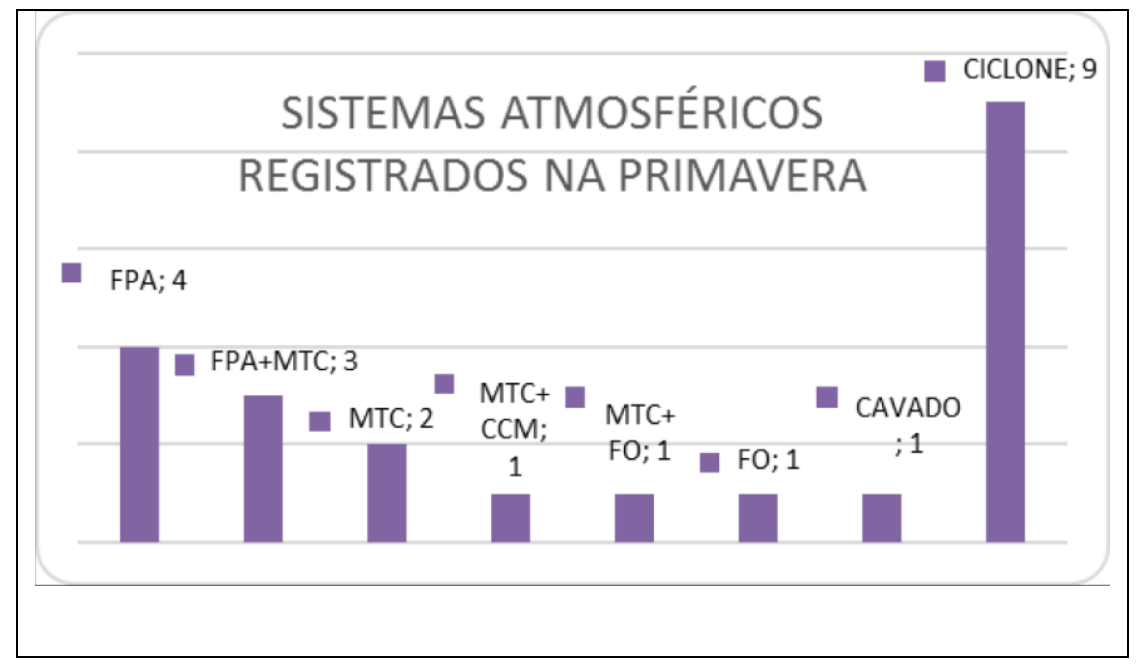

Org.: Writzl, L. (2019)

Figura 11 - Sistemas atmosféricos registrados no outono

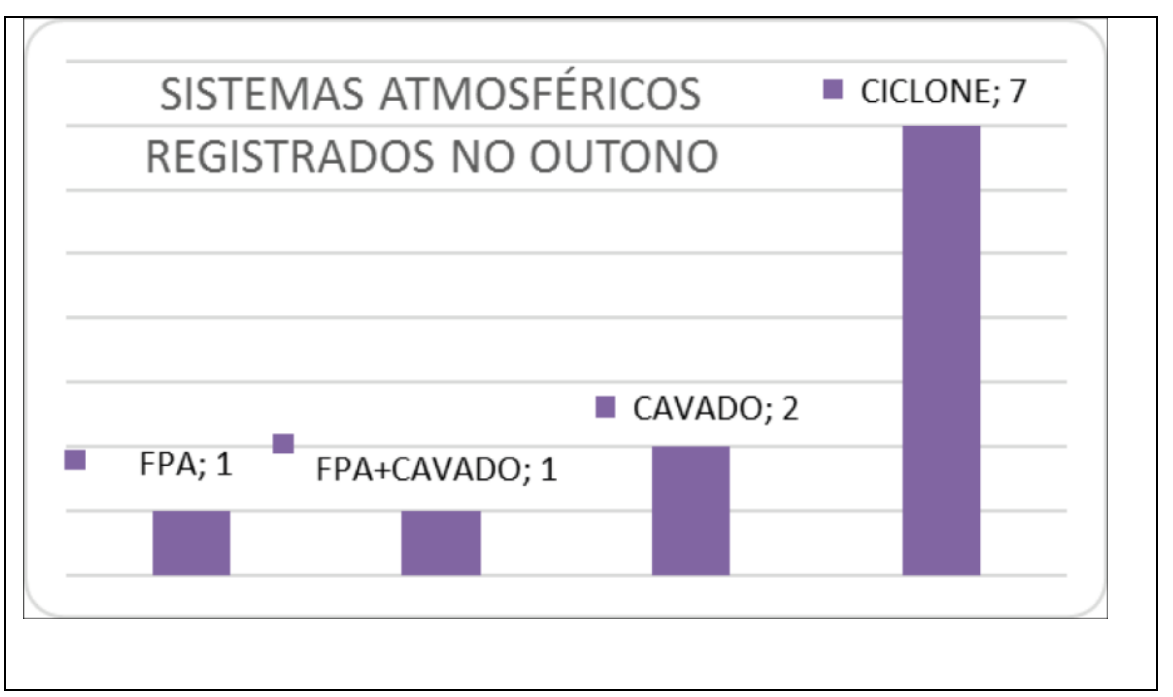

Org.: Writzl, L. (2019)

\section{CONCLUSÕES}

Visto a carência de pesquisas relacionadas a esta área de estudo, esse trabalho proporcionou o entendimento de que os tornados são frequentes no Brasil, mas principalmente no Rio Grande do Sul. Através de diversas fontes, foi possível reunir as ocorrências dos tornados registrados no estado, e construir uma tabela com os principais dados para o período de análise. Os tornados ocorrem em praticamente 
todas as regiões do estado, o que falta em muitas ocasiões são registros diários das ocorrências em cidades menos populosas, para resultados mais precisos.

O estudo proporcionou uma visão ampla para as perspectivas futuras, onde é importante tomar conhecimento sobre esses eventos que ocorrem frequentemente no Rio Grande do Sul e na maioria das vezes não são registrados. É de grande importância que a população conheça mais sobre esses fenômenos, fortalecendo essa área de estudo principalmente no ensino básico, para que sejam minimizados os danos, pois eles afetam as variadas formas de vida presentes nos locais em que ocorrem.

\section{REFERÊNCIAS}

BECK AT, VERZENHASSI, CC. Reliability Based Risk Optimization. In: 4th International ASRANet Colloquium, Atenas, Grecia: ASRANet Ltd., 2008.

BURROUGH PA, McDONNELL RA. Principles of Geographical Information Systems. Nova York: Oxford University Press, 1998

CANDIDO DH. tornados e trombas d'água no Brasil: Desenvolvimento de um modelo e proposta de escala de avaliação de danos. 2012. 230 f. Tese (Doutorado em Geociências) - Universidade Estadual de Campinas. 2012.

CARTAS SINÓTICAS. Centro de Hidrografia da Marinha. Disponível em: https://www.marinha.mil.br/chm/dados-do-smm-cartas-sinoticas/cartas-sinoticas. Acesso em: Junho de 2019.

COPPOCK JT. GIS and natural hazards: an overview form a GIS perspective. In: CARRARA, A.; GUZZETTI, F. (Ed.) Geographical information systems in assessing natural hazards. Dordrecht: Kluwer Academic Publishers, 1995.

DYER RA. A review of tornado activity in Brazil. In: International Society for Photogrammetry and Remote Sensing, 7., 1994, Rio de Janeiro. Anais... São José dos Campos: INPE, v. 30 part 7a, 1994, p.203-213.

IBGE. Bases e referencias. Disponível em: https://mapas.ibge.gov.br/bases-ereferenciais/bases-cartograficas/malhas-digitais. Acesso em: agosto de 2019.

IENSSE AC. CIRCULAÇÃO ATMOSFÉRICA REGIONAL E A OCORRÊNCIA DE TORNADOS E TROMBAS D'ÁGUA NO RIO GRANDE DO SUL, NO PERÍODO DE 2001 A 2015. 70p. (Trabalho de Graduação) - Universidade Federal de Santa Maria. 2015. 
NASCIMENTO EL. Previsão de tempestades severas utilizando-se parâmetros convectivos e modelos de mesoescala: uma estratégia operacional adotável no Brasil. Revista Brasileira de Meteorologia, São José Dos Campos, v. 20, n. 1, p.121-140, 15 fev. 2005.

NIMER E., "Clima Região Sul". in: Geografia do Brasil, v 2. IBGE, Rio de Janeiro, 1990

RECKZIEGEL BW. Levantamento dos desastres desencadeados por eventos naturais adversos no Estado do Rio Grande do Sul no período de 1980 a 2005. 2007. 370 f. Dissertação (Mestrado em Geografia) - Universidade Federal de Santa Maria, Santa Maria, 2007.

REDEMET. Rede de Meteorologia do Comando da Aeronáutica. Disponível em: https://www.redemet.aer.mil.br/index.php. Acesso em: agosto de 2019.

SLEIMAN J. Veranicos ocorridos na porção noroeste do Estado do Rio Grande do Sul entre 1978 e 2005 e sua associação às condições climáticas da atmosfera. 164p Dissertação (Mestre em Geografia) - Universidade de São Paulo. 2008.

WOLLMANN CA, GALVANI, E. Caracterização Climática Regional do Rio Grande do Sul: Dos Estudos Estáticos Ao Entendimento Da Gênese. Revista Brasileira de Climatologia. Ano 8 - Vol 11 jul/dez. 2012. 